\title{
PMK: Medidas Válidas para a Predição do Desempenho no Trabalho?
}

\author{
MKP: Are there Validity Measures to Predict Job Performance?
}

\author{
Alina Gomide Vasconcelos*, Jáder dos Reis Sampaio \& Elizabeth Nascimento \\ Universidade Federal de Minas Gerais, Belo Horizonte, Brasil
}

\begin{abstract}
Resumo
O Psicodiagnóstico Miocinético (PMK) tem sido considerado uma técnica útil na avaliação da personalidade pelos profissionais, embora sejam escassas as investigações que comprovem sua utilidade do ponto de vista aplicado. O objetivo do estudo foi investigar a validade preditiva das dimensões do PMK em relação ao desempenho no trabalho em uma organização estadual. Participaram deste estudo 248 indivíduos destros, com idade média de 22,67 ( $D P=3,05)$ anos, sendo 83,90\% do sexo masculino. Os resultados das associações univariadas entre os 66 traçados do PMK e o desempenho, embora significativos, foram fracos. As associações encontradas não se sustentaram na análise de regressão logística. Esses resultados indicaram que as dimensões teóricas do PMK, tal como preconizadas no manual do instrumento, sendo elas investigados isoladas ou em conjunto, não foram relevantes para predizer o desempenho no trabalho dos funcionários da organização estudada. Discutem-se os cuidados metodológicos e os limites da presente investigação.

Palavras-chave: Validade de critério, PMK, desempenho no trabalho.
\end{abstract}

\begin{abstract}
Myokinetic Psychodiagnosis (MKP) has been considered a useful instrument by the Brazilian professional community, although there are few validity investigations about its psychometrics properties. The aim of this study was to investigate the predictive validity of MKP measures in relation to job performance. The sample comprised 248 right-handed individuals, with a mean age of 22.67 years $(S D=3.05), 83.90 \%$ are male and all of them had finished high school. Preliminarily, exploratory factor analyses were conducted to obtain factors corresponding to the theoretical dimensions of the instrument. Results of univariate associations between measures were significant, but weak. These associations were not sustained in logistic regression analysis. The results indicated that MKP theoretical dimensions were not relevant to predict job performance in the studied institution.

Keywords: Validity, MKP, job performance.
\end{abstract}

No contexto da Psicometria, validade se refere ao grau em que as evidências acumuladas por meio de vários estudos indicam que os escores de um instrumento oferecem informações legítimas sobre o construto psicológico que se pretende avaliar (American Educational Research Association [AERA], American Psychological Association [APA], \& National Council on Measurement in Education [NCME], 1999). A abordagem atual sobre a validade propõe um modelo no qual são integrados os vários tipos conhecidos de validade, incluindo o modelo trinitário proposto por Cronbach e Meehl (1955), composto pelas categorias validade de construto, critério e de conteúdo. $\mathrm{O}$ enfoque atual enfatiza que a validade de construto deve

* Endereço para correspondência: Departamento de Psicologia, Laboratório das Diferenças Individuais, Faculdade de Filosofia e Ciências Humanas, Universidade Federal de Minas Gerais, Av. Presidente Antônio Carlos, 6627, Cidade Universitária, Pampulha, Belo Horizonte, MG, Brasil 31270-901.E-mail: alinagomide@gmail.com ser entendida como um conceito integrado e multifacetado resultante das várias fontes de evidências de validade e não mais como uma categoria de validade (AERA et al., 1999; Cronbach, 1949; Embretson, 1983). Nesse sentido, tem-se que as fontes de evidências de validade contribuem com informações sobre a validade de construto dos escores do instrumento e podem ser relacionadas: (a) ao conteúdo do teste, (b) aos processos de resposta, (c) à estrutura interna, (d) às relações dos escores do teste com outras variáveis e, (e) às consequências do uso do teste (AERA et al., 1999). Esta integração teve como intuito enfatizar que quaisquer destes procedimentos contribuem para a definição teórica do construto psicológico e para a compreensão de suas inter-relações com as medidas de variáveis observáveis e com os construtos.

Pode-se descrever de forma simplificada as relações entre as variáveis envolvidas no processo reunir evidências de validade de construto, a saber, os construtos e medidas, os construtos e medidas de critério (Figura 1; Binning \& 
Barrett, 1989; Society for Industrial and Organizational Psychology [SIOP], 2003). Esse modelo, que se mostra útil para os estudos de validade no contexto do trabalho, representa graficamente as cinco possíveis relações entre construtos e suas respectivas medidas.

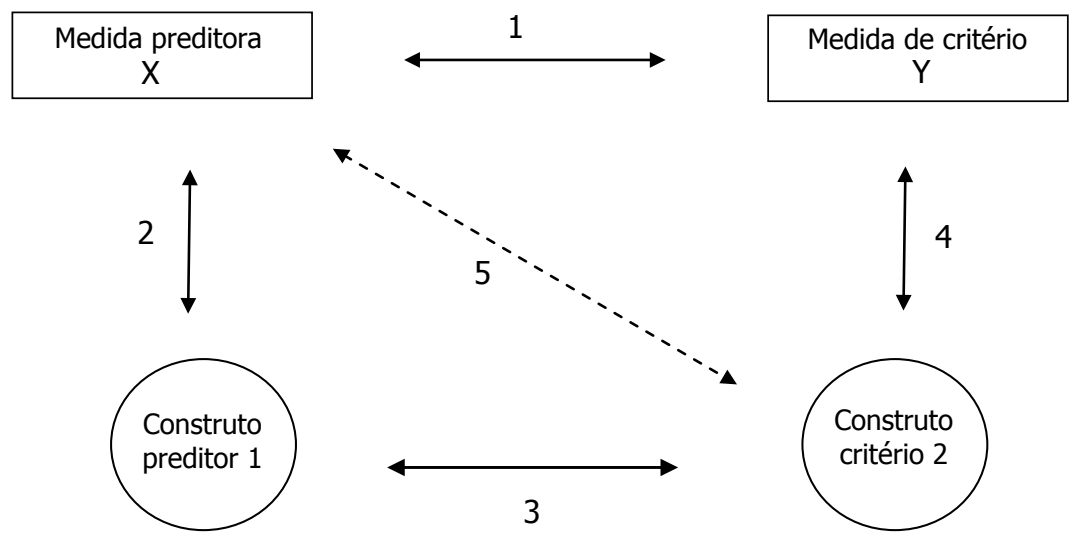

Figura 1. Relações entre construtos e medidas - Adaptado de Binning e Barrett (1989) e SIOP, (2003).

Os preditores são medidas obtidas por meio de técnicas de seleção como, por exemplo, os escores nos testes psicológicos. Já, dentre as medidas de critério no contexto do trabalho, podem ser utilizados os indicadores do desempenho dos indivíduos no treinamento ou na função ocupacional. Na Figura 1, a seta identificada com o número 3 representa as evidências de validade de conteúdo. A seta identificada com o número 1 refere-se aos estudos de validade das relações dos escores do teste com outras variáveis. Esta associação é a única que pode ser testada de modo empírico (Schmitt \& Landy, 1993). Já as setas numeradas com os números 2 e 4 representam a validade de conteúdo, que legitimam a associação entre os itens do instrumento e o construto de interesse. Finalmente, a seta 5 refere-se aos estudos relacionados à validade de conteúdo, nos quais se busca identificar os componentes do construto que estão sendo avaliados pelas medidas preditoras. As setas supracitadas são relevantes para reunir evidências da validade de construto em um sentido mais amplo. No entanto, o pesquisador pode dar maior ênfase a uma delas, de acordo com a etapa do processo de construção e validação de um instrumento.

Dentre as fontes de evidência de validade, os estudos das relações dos escores do teste com outras variáveis são de grande interesse para os pesquisadores e profissionais na seleção de pessoas, uma vez que o uso de instrumentos psicológicos visa antever o comportamento futuro da pessoa na tarefa (Hough \& Oswald, 2000; Schmidt \& Hunter, 1998; SIOP, 2003; Spector, 2004). Nesse contexto, as evidências de validade relacionadas ao critério podem ser entendidas como o grau em que os escores do teste estão correlacionados com os indicadores comportamentais da adaptação da pessoa ao ambiente e ao cargo para ao qual foi selecionada, considerando os contextos de trabalho específicos (SIOP, 2003). Delimitado o indicador comportamental que será estudado (critério), espera-se que as medidas psicológicas (preditoras) sejam capazes de distinguir indivíduos com desempenho superior e indivíduos com desempenho inferior para que as interpretações decorrentes consideradas válidas. Os indicadores de desempenho no contexto do trabalho podem ser de natureza objetiva e geralmente são coletados em registros administrativos da organização a respeito do comportamento de seus funcionários, por exemplo, número de faltas, número de acidentes, número de transgressões disciplinares, número de licenças médicas, número de premiações, progressão salarial e progressão de carreira, medidas de produtividade, entre outras medidas relevantes para avaliar o desempenho do indivíduo na função e contextos específicos (Spector, 2004).

No que diz respeito aos preditores do desempenho no trabalho, uma ampla variedade de fatores individuais pode influenciar o comportamento no treinamento e no trabalho. Dentre eles, a habilidade cognitiva e os traços de personalidade tradicionalmente são os mais investigados como preditores do desempenho no trabalho. Estudos reportados na literatura sobre a validade preditiva dos escores dos testes de inteligência identificaram um relacionamento direto entre esse construto e o desempenho (Hough \& Oswald, 2000; Robertson \& Smith, 2001; Smith, 1994) tanto no treinamento (Hunter \& Hunter, 1984; Schmidt \& Hunter, 1998) quanto no trabalho (Kuncel, Hezlett, \& Ones, 2004; Mount, Barrick, \& Strauss, 1999).

Já os estudos sobre a validade preditiva das medidas de traços de personalidade no ambiente de trabalho apresentam resultados contraditórios e inconsistentes (Morgeson et al., 2007a; Tett \& Christiansen, 2007). Por isso, ainda não existe consenso na literatura sobre a qualidade preditiva dessas medidas, apesar de serem utilizadas com frequência na seleção de pessoas. A validade da testagem psicológica na área de seleção de pessoas sempre foi acompanhada por discussões sobre as implicações éticas e a identificação dos fatores individuais específicos relevantes ao desempenho futuro na função, principalmente, 
de traços de personalidade. Essas questões podem estar relacionadas ao fato de ainda existirem poucos estudos empíricos que investiguem sistematicamente a validade dos testes psicológicos como preditores do desempenho do indivíduo no trabalho (Schmidt \& Hunter, 1998, 2004) e também ao fato de que são encontradas correlações de baixa magnitude entre essas medidas (Sampaio, 1998; Schmidt \& Hunter, 2004).

A literatura aponta que a relação entre os aspectos de personalidade e as medidas de desempenho é complexa (Smith, 1994; Spector, 2004), por isso, o alcance de coeficientes de validade satisfatórios obtidos a partir de relações diretas entre as medidas desses dois construtos depende de uma cuidadosa seleção das variáveis individuais baseada na compreensão teórica e prática dos indicadores de desempenho (Morgeson et al., 2007a). Borman e Motowidlo (1997) propuseram que as características psicológicas, habilidades cognitivas e personalidade, apresentam poder de predição diferenciado em relação às dimensões do desempenho no trabalho. A hipótese é de que as características de personalidade são melhores preditores do desempenho contextual que se refere a comportamentos relevantes para a manutenção ou melhoria do ambiente institucional, social e psicológico no qual as atividades prescritas são desenvolvidas (Motowidlo, Borman, \& Schmit, 1997). Exemplos desses comportamentos incluem auxiliar e cooperar com os outros, ser voluntário para fazer além do que é o mínino prescrito necessário, persistir para completar a tarefa, seguir as regras mesmo quando pessoalmente inconvenientes e apoiar e defender os objetivos da instituição. Já as habilidades cognitivas afetariam o desempenho na tarefa, ou seja, as atividades prescritas para a função que contribuem diretamente ou que oferecem suporte para o alcance do sucesso esperado pela instituição. Essa proposta é convergente com a afirmação de Salgado (1997) a respeito de que diferentes conjuntos de variáveis de personalidade são úteis para predizer aspectos específicos do desempenho, e, além disso, interagem com a habilidade cognitiva de acordo com a função ocupacional. Estas especificidades nas relações entre as medidas corroboram a importância dos procedimentos de análise do cargo e do desenvolvimento de um perfil psicológico profissional para basear a escolha dos instrumentos de seleção de pessoas.

$\mathrm{Na}$ literatura brasileira, foram encontrados apenas quatro estudos a respeito da validade dos testes psicológicos que avaliam a personalidade no contexto do trabalho (Baumgartl \& Primi, 2006; Dias, 2005; Freitas, 2004; T. S. Vasconcelos, 2005). Dentre esses estudos, três utilizaram medidas de auto-relato para avaliação da personalidade e apenas um contemplou o Psicodiagnóstico Miocinético (PMK), objeto de investigação da presente pesquisa. Freitas (2004) desenvolveu um estudo para verificar a validade preditiva das medidas de personalidade por meio do Inventário Fatorial da Personalidade - Revisado (IFP-R) e do Wartegg em relação ao desempenho no treinamento em uma organização militar. Os resultados de regressão hierárquica indicaram que os cinco escores fatoriais, formados a partir da reunião dos fatores de personalidade avaliados pelo IFP-R, explicaram $2 \%$ da variância nas notas nas disciplinas físicas. $\mathrm{O}$ poder de explicação das variáveis psicológicas pode ser considerado baixo e não se manteve quando as variáveis sócio-demográficas (idade, escolaridade, estado civil, etnia, religião) e cognitivas foram inseridas no modelo. Os coeficientes de regressão dos escores fatoriais formados a partir dos instrumentos de personalidade foram menos significativos quando se incluiu os escores do Wartegg (Freitas, 2004). Por isso, as análises foram realizadas sem considerar tais escores.

Com base no modelo de personalidade dos Cinco Fatores da Personalidade, T. S. Vasconcelos (2005) realizou um estudo com uma amostra de policiais civis já admitidos na instituição. Os resultados indicaram evidências de validade preditiva do Inventário Reduzido dos Cinco Fatores de Personalidade (ICFP) em relação a medidas no treinamento para a execução da função. Dentre as medidas de personalidade, o fator Conscienciosidade se mostrou como o preditor importante das médias nas disciplinas ministradas no treinamento no modelo de regressão hierárquico $\left(R^{2}=0,10\right)$. Trata-se de uma associação fraca sendo o resultado condizente com pesquisas anteriores (Morgeson et al., 2007a; Tett \& Christiansen, 2007). Já o estudo conduzido por Dias (2005), no qual se investigou a relação entre as medidas de personalidade obtidas por meio 16 Fatores de Personalidade (16PF) e o absenteísmo no trabalho, os resultados indicaram correlações fracas ou nulas entre os fatores de segunda ordem investigados por meio desse instrumento e a variável dependente absenteísmo. Apenas o fator auto-controle correlacionou-se significativamente com a mesma, no entanto, a magnitude da associação foi baixa $(r=-0,10)$.

Embora o PMK seja considerado uma técnica bastante útil na avaliação da personalidade no Brasil pela comunidade profissional, as investigações que comprovem sua utilidade do ponto de vista aplicado são escassas (Sisto, 2010). Conforme exposto anteriormente, localizou-se apenas o estudo de Baumgartl e Primi (2006) sobre a validade referente a critério das dimensões de personalidade obtidas por meio do PMK no contexto do trabalho. As medidas de desempenho foram a nota na avaliação de desempenho, o número de acidentes de trabalho e a produtividade de profissionais de uma empresa de energia elétrica. As análises de correlação indicaram associação positiva e significativa entre o fator denominado agressividade e o número total de acidentes no trabalho $(r=0,28 ; p<0,05)$. Trata-se de uma associação de magnitude baixa e indicou que quanto mais o indivíduo experiente dirige sua agressividade para o meio, mais propenso ele está de sofrer acidentes de serviço. Contudo, a variável agressividade explicou apenas $8 \%$ da variância observada nos acidentes de trabalho $\left(R^{2}=0,08\right)$. Nesse estudo, é importante observar que a dimensão agressividade foi heterogênea quanto aos traçados que a compõem, pois incluiu traçados relacionados aos fatores teóricos advindos da reação vivencial, agressividade e 
tônus vital. Além disso, obteve-se associação positiva e significativa entre o fator denominado impulsividade e a produtividade dos funcionários $(r=0,69 ; p<0,01)$. Esse resultado indicou que funcionários mais impulsivos tendem a obter maiores índices de produtividade. A dimensão impulsividade foi formada por nove traçados relativos aos fatores teóricos tônus vital, agressividade, reação vivencial, predomínio tensional e emotividade. A autora do estudo reportado considerou incoerente esse resultado, tendo em vista a fundamentação teórica do instrumento e não ofereceu hipóteses alternativas para explicar a associação obtida.

$\mathrm{Na}$ literatura internacional, a ampla discussão sobre a validade preditiva dos testes de personalidade no contexto do trabalho está pautada maciçamente nos resultados gerados a partir de instrumentos de autorrelato (Borman \& Motowidlo, 1997; Morgeson et al., 2007a; Tett \& Christiansen, 2007), tendência também presente no contexto brasileiro. Morgeson et al. (2007a) concluíram que, apesar da utilização dos testes de autorrelato para avaliação da personalidade ser fundamental para tomada de decisão na seleção de pessoas, não há evidências suficientes de que eles são bons preditores isolados do desempenho no trabalho. Tal conclusão reforça a importância de investigações sobre a validade preditiva das medidas psicológicas em relação ao desempenho no trabalho, inclusive de medidas expressivas de personalidade.

Considerando-se a ampla utilização do PMK no contexto organizacional brasileiro, o objetivo do presente estudo foi investigar a validade preditiva dos traçados desse instrumento em relação ao desempenho no trabalho em uma organização pública do estado de Minas Gerais. Tendo como base o modelo apresentado sobre o levantamento de evidências de validade (Binning \& Barrett, 1989; SIOP, 2003), a proposta teórica de Mira e Lopez (Galland de Mira, 2002) e a análise do perfil profissiográfico do cargo conduzida por A. G. Vasconcelos (2010), a hipótese adotada foi que trabalhadores que apresentassem controle sobre a expressão emocional e comportamental, índices de tônus vital e agressividade variando entre normal a aumentado tenderiam a alcançar medidas de desempenho no trabalho mais satisfatórias. No entanto, em caráter exploratório, todas as dimensões de personalidade avaliadas pelo PMK foram incluídas nas análises e investigou-se a associação entre elas e o desempenho no trabalho.

\section{Método}

\section{Participantes}

Participaram do estudo 248 indivíduos destros, admitidos na organização por meio do concurso público. A opção pelos indivíduos com lateralidade destra deveu-se ao fato de que as normas de interpretação dos escores do PMK foram desenvolvidas com base em uma amostra que não contemplou indivíduos canhotos (Galland de Mira, 2002). A média de idade dos participantes foi de 22,67 $(D P=3,05)$ anos com variação entre 18 e 30 anos. A maioria era do sexo masculino $(83,90 \%)$ e todos apresentavam ensino médio completo. Em relação ao estado civil, 92,30\% eram solteiros.

\section{Instrumentos}

O Psicodiagnóstico Miocinético foi desenvolvido por Mira y Lopes com o objetivo de investigar os aspectos estruturais e reacionais da personalidade relacionados às dimensões Tônus Vital, Agressividade, Dimensão Tensional, Reação Vivencial, Emotividade e Predomínio Tensional por meio de respostas psicomotoras (Galland de Mira, 2002). A aplicação completa do teste, composto por seis folhas, é individual e não possui tempo limitado e deve ser realizada em duas sessões com intervalo mínimo de 24 horas e intervalo máximo de sete dias (Galland de Mira, 2002). Quantitativamente, são obtidos 76 traçados que oferecem informações para os diferentes traços de personalidade avaliados pelo instrumento. Em relação à apuração dos resultados, têm-se disponíveis tabelas de normas com relação ao sexo e os escores brutos são convertidos em tetrons na classificação final. Outras tendências psicológicas relacionadas ao tipo de movimento podem ser identificadas por meio da análise qualitativa dos traçados, tais como a excitabilidade ou inibição, ansiedade ou angústia, nível ideomotor, impulsividade e outros aspectos decorrentes da avaliação qualitativa dos traçados do indivíduo (Galland de Mira, 2002). Nesse estudo, foi utilizada a versão reduzida do PMK que inclui a aplicação dos traçados das seis folhas, exceto as configurações não-lineares referentes aos círculos e aos elos ascendentes e descendentes. Diante dessa redução dos traçados aplicados, são obtidas 66 medidas. No Brasil, é comum a utilização do PMK reduzido tanto nos processos seletivos quanto nos exames psicotécnicos para a obtenção da carteira nacional de habilitação. No entanto, esse formato de aplicação é expressamente desaconselhado no manual técnico do instrumento por ser considerado "uma mutilação" dos resultados do teste (Galland de Mira, 2002, p. 48). Quando na realização do estudo, constava no sistema SATEPSI a aprovação do PMK.

As medidas de desempenho contempladas foram definidas a partir da revisão de literatura e se referem a quatro medidas de recompensa e cinco medidas sobre comunicações disciplinares no contexto do trabalho (Borman \& Motowidlo, 1997; Spector, 2004). As recompensas são benefícios materiais e distinções concedidas aos indivíduos em razão de atos meritórios, serviços relevantes considerando os princípios de proporcionalidade, individualidade, oportunidade, merecimento (documento oficial da organização). As recompensas foram classificadas de acordo com os eventos geradores em quatro categorias pelos autores desta pesquisa: (a) apresentar desempenho eficaz e eficiente por meio do emprego de capacidade técnica em benefício do serviço; (b) colaborar de maneira espontânea com a efetividade dos resultados pretendidos; (c) participar em eventos; (d) observar as normas e as prescrições regulamentares. Já as comunicações disciplinares se referem a uma formalização escrita acerca de ato ou fato que ofenda aos princípios da ética e o não cumprimento dos deveres 
inerentes à função (documento oficial da organização). O julgamento da transgressão é realizado por comissão instaurada para esse fim e de acordo com os critérios discriminados no Código de Ética (documento oficial da organização). As comunicações disciplinares foram classificadas de acordo com os eventos geradores em cinco categorias: (a) faltar ou chegar atrasado ao serviço injustificadamente o que compromete a execução dos serviços e atividades; (b) ter desempenho insuficiente na execução das tarefas; (c) apresentar postura desrespeitosa; (d) negligenciar o cumprimento de normas, prazos e tarefas; (e) ter postura incompatível com os princípios e valores da organização e que não se encaixa nas categorias descritas acima (por exemplo, roubos e extravios).

\section{Procedimentos de Coleta dos Dados}

O estudo foi previamente aprovado junto ao Comitê de Ética da Universidade Federal de Minas Gerais. Os dados foram obtidos por meio de análise documental que incluiu a consulta aos arquivos físicos do Serviço de Psicologia da organização a fim de se obter os escores do PMK e as variáveis de desempenho no trabalho.

\section{Procedimentos de Análise dos Dados}

Inicialmente, foi realizada uma categorização dos eventos geradores de recompensas e comunicações disciplinares pelos autores do presente trabalho tendo como base documentos da organização e a literatura sobre desempenho no trabalho (Borman \& Motowidlo, 1997). A alta frequência de um evento gerador de recompensa ou comunicação disciplinar e a relevância teórica de determinados eventos foram considerados como critérios para a formação das categorias, dando origem a quatro categorias relacionadas às recompensas e cinco relacionadas a eventos geradores de comunicação disciplinar conforme já apresentadas.

Em relação às medidas do $\mathrm{PMK}$, foram utilizados os fatores extraídos pela Análise Fatorial Exploratória. O conjunto de traçados de cada uma das 6 dimensões teóricas propostas por Mira y Lopes foram analisados por meio da Análise Fatorial Exploratória (AFE) separadamente. Em cada dimensão analisada, identificou-se a presença de 2 fatores específicos diretamente relacionados aos traçados de cada uma das mãos. Como exemplo, o fator teórico tônus vital ficou representado pelos traçados preconizados pelo manual do teste. No entanto, dois fatores específicos foram identificados, sendo o primeiro fator correspondente aos traçados da mão direita e um segundo aos traçados da mão esquerda. Foram geradas 10 medidas (escores fatoriais) resultantes de 2 fatores (mão direita e mão esquerda) para cinco dimensões teóricas (tônus vital, emotividade, agressividade, reação vivencial e predomínio tensional). A única exceção ocorreu para a dimensão tensional em que as medidas de ambas as mãos foram reunidas. Ao final, 11 medidas foram utilizadas. A descrição detalhada dos procedimentos realizados a respeito da AFE está disponível em A. G. Vasconcelos (2010; A. G. Vasconcelos, Nascimento, \& Sampaio, 2011). Enfatiza-se que os fatores latentes obtidos correspondem às dimensões de personalidade propostas por Mira y Lopes (Galland de Mira, 2002).

Em seguida, os escores das dimensões do PMK obtidos foram codificados em categorias ordinais com base nas referências apresentadas no manual técnico para a interpretação das medidas do instrumento. Com base nestes pontos de corte, nesse estudo, delimitaram-se 5 categorias, formadas a partir dos valores contínuos dos escores fatoriais, a saber normal, diminuída, aumentada, patológico diminuído, patológico aumentado. As denominações e os escores limites das categorias foram: (a) normal: formada a partir dos escores fatoriais médios, correspondente a $-1 D P$ a $+1 D P$; (b) diminuída: formada a partir do intervalo de valores correspondentes aos escores fatoriais diminuídos $(-2 D P$ a $-1 D P)$; (c) aumentada: referente aos escores fatoriais aumentados $(+1 D P$ a $+2 D P)$; (d) patológica diminuída: constituída pelos escores fatoriais situados na zona patológica diminuída (abaixo de -2DP); (e) patológica aumentada: composta pelos escores fatoriais localizados na zona patológica aumentada (acima de $+2 D P$ ). As análises subsequentes que incluem os traçados do PMK foram realizadas com base nas categorias obtidas.

Foram realizadas análises estatísticas descritivas a fim de caracterizar a amostra e as variáveis do estudo. Para investigar o grau de associação entre as medidas categoriais do PMK (categorias dos escores fatoriais) e as variáveis de desempenho, mensuradas em nível nominal, foi utilizado o coeficiente $\mathrm{V}$ de Cramér. A interpretação do coeficiente indica a força da associação entre as variáveis que pode variar entre 0 a 1 , não admitindo valores negativos. Infelizmente, ele não fornece informação sobre a direção da associação como o coeficiente de correlação de Pearson. Os valores significativos próximos de 0 indicam baixa associação entre as duas variáveis e valores significativos próximos de 1, fortes associações (Siegel \& Castellan, 2006). A significância estatística do V de Cramér pode ser obtida e corresponde ao mesmo valor da significância do qui-quadrado ao qual está relacionado. Finalmente, modelos multivariados foram obtidos por meio da técnica de análise de regressão logística bivariada tendo como variável dependente a presença de recompensas ou punições. Os modelos de regressão logística foram construídos separadamente para cada variável de desempenho no trabalho (variáveis dependentes) que apresentou pelo menos uma correlação significativa com uma das dimensões do PMK, considerando-se o nível de significância de 25\% (Ayçaguer \& Utra, 2004; Hosmer \& Lemeshow, 2000). Para as análises dos demais dados, adotou nível de significância de 5\%.

\section{Resultados}

As estatísticas descritivas para os escores ordinais nas dimensões do PMK são apresentadas na Tabela 1. Nota-se que a maioria dos participantes (aproximadamente 70\%) apresentou escores dentro da zona de normalidade no fator tônus vital do PMK e em ambas as mãos. 
Tabela 1

Estatísticas Descritivas a Frequência de Indivíduos nas Dimensões Teóricas do PMK (N=248)

Categorias dos escores fatoriais * Fatores teóricos do PMK mensurados em nível ordinal

\begin{tabular}{|c|c|c|c|c|}
\hline & TV - MD & TV - ME & AGR - MD & AGR - ME \\
\hline & $F(\%)$ & $F(\%)$ & $F(\%)$ & $F(\%)$ \\
\hline Patológico diminuído & $174(70,16)$ & $178(71,77)$ & $176(70,97)$ & $174(70,16)$ \\
\hline Diminuído & $33(13,31)$ & $26(10,48)$ & $31(12,50)$ & $33(13,31)$ \\
\hline Médios & $27(10,89)$ & $31(12,50)$ & $27(10,89)$ & $29(11,69)$ \\
\hline Aumentado & $7(2,82)$ & $8(3,23)$ & $6(2,42)$ & $4(1,61)$ \\
\hline \multirow[t]{3}{*}{ Patológico aumentado } & $7(2,82)$ & $5(2,02)$ & $8(3,23)$ & $8(3,23)$ \\
\hline & RV - MD & $\mathrm{RV}-\mathrm{ME}$ & EMO - MD & $\mathrm{EMO}-\mathrm{ME}$ \\
\hline & $F(\%)$ & $F(\%)$ & $F(\%)$ & $F(\%)$ \\
\hline Patológico diminuído & $180(72,58)$ & $171(68,95)$ & $187(75,40)$ & $183(73,79)$ \\
\hline Diminuído & $27(10,89)$ & $35(14,11)$ & $21(8,47)$ & $24(9,68)$ \\
\hline Médios & $26(10,48)$ & $33(13,31)$ & $25(10,08)$ & $28(11,29)$ \\
\hline Aumentado & $8(3,22)$ & $3(1,21)$ & $1(0,40)$ & $3(1,21)$ \\
\hline \multirow[t]{3}{*}{ Patológico aumentado } & $7(2,82)$ & $6(2,42)$ & $14(5,66)$ & $10(4,03)$ \\
\hline & PRE TEN - MD & PRE TEN - ME & DIM TEN - ambas mãos & \\
\hline & $F(\%)$ & $F(\%)$ & $F(\%)$ & \\
\hline Patológico diminuído & $171(68,96)$ & $190(76,61)$ & $176(70,97)$ & \\
\hline Diminuído & $32(12,90)$ & $27(10,89)$ & $31(12,50)$ & \\
\hline Médios & $31(12,50)$ & $15(6,05)$ & $28(11,29)$ & \\
\hline Aumentado & $7(2,82)$ & $3(1,21)$ & $6(2,42)$ & \\
\hline Patológico aumentado & $7(2,82)$ & $13(5,24)$ & $7(2,82)$ & \\
\hline
\end{tabular}

Nota. Legenda: TV: Tônus vital; AGR: Agressividade; RV: Reação vivencial; EMO: Emotividade; PRE TEN: Predomínio tensional; DIM TEN: Dimensão tensional; MD: Mão direita; ME: Mão esquerda.

No total, foram obtidos 187 coeficientes de $\mathrm{V}$ de Cramér (11 dimensões do PMK X 17 medidas de desempenho no trabalho). Todas as associações foram de fraca magnitude com variação da amplitude entre 0,03 e 0,37 . Além disso, $74,87 \%$ (140) das associações não foram significativas, $18,62 \%$ (35) foram significativas considerando-se o nível de significância igual ou menor que $25 \%$, finalmente, apenas 5,38\% (12) significativas a menor que 5\%. Dentre as medidas de personalidade utilizadas, apenas nove dimensões do PMK apresentaram associação significativa a $25 \%$ com pelo menos uma medida de desempenho. Diante desses resultados, optou-se por realizar a análise de regressão múltipla para se investigar se as associações entre as nove medidas de personalidade e de desempenho e se manteriam em uma análise conjunta. Logo, as asso- ciações significativas foram testadas em nove modelos de regressão logística binária conforme a Tabela 2 , em que são apresentadas as variáveis dependentes e independentes que compuseram os modelos de regressão logística, identificadas a partir da análise univariada.

Em geral, a avaliação global do ajuste de cada um dos modelos a partir da inspeção visual da tabela de classificação indicou uma baixa concordância entre frequências esperadas e observadas para as condições da variável resposta, apesar de existir um número satisfatório de casos nas duas condições da variável resposta, o que permite concluir que o mau ajuste não pode ser explicado por um viés da amostra e sim devido à ausência de relação preditiva entre as variáveis. Além disso, a avaliação do ajuste geral dos modelos indicou variação significativa 
Vasconcelos, A. G., Sampaio, J. R. \& Nascimento, E. (2013). PMK: Medidas Válidas para a Predição do Desempenho no Trabalho?

Tabela 2

Variáveis Dependentes e Independentes dos Modelos de Regressão Logística e Magnitude das Associações Significativa Univariadas (V de Cramer)

\begin{tabular}{|c|c|c|c|}
\hline Modelo & Variável dependente & Variáveis independentes & V de Cramer \\
\hline Modelo 1 & Comunicação disciplinar & Reação vivencial - ME & $0,23 * *$ \\
\hline \multirow[t]{4}{*}{ Modelo 2} & \multirow{4}{*}{$\begin{array}{l}\text { Comunicação disciplinar por faltar } \\
\text { ao trabalho }\end{array}$} & Emotividade - MD & $0,21^{*}$ \\
\hline & & Agressividade - ME & $0,21 * *$ \\
\hline & & Reação vivencial - ME & $0,20^{*}$ \\
\hline & & Dimensão tensional - DUAS & $0,15 * * *$ \\
\hline \multirow[t]{3}{*}{ Modelo 3} & \multirow{3}{*}{$\begin{array}{l}\text { Comunicação disciplinar por negligenciar } \\
\text { o cumprimento de normas }\end{array}$} & Tônus vital - MD & $0,14 * * *$ \\
\hline & & Agressividade - ME & $0,14 * * *$ \\
\hline & & Predomínio tensional - ME & $0,09 * * *$ \\
\hline \multirow[t]{3}{*}{ Modelo 4} & \multirow{3}{*}{$\begin{array}{l}\text { Comunicação disciplinar por chegar } \\
\text { injustificadamente atrasado }\end{array}$} & Predomínio tensional - MD & $0,15^{* * *}$ \\
\hline & & Emotividade - ME & $0,17 * * *$ \\
\hline & & Dimensão tensional - DUAS & $0,15^{* * *}$ \\
\hline \multirow[t]{3}{*}{ Modelo 5} & \multirow[t]{3}{*}{ Recompensas } & Tônus vital - MD & $0,17 * * *$ \\
\hline & & Tônus vital - ME & $0,18^{* * *}$ \\
\hline & & Predomínio tensional - ME & $0,17 * * *$ \\
\hline \multirow[t]{3}{*}{ Modelo 6} & \multirow[t]{3}{*}{ Recompensa por participação em eventos } & Agressividade - ME & $0,22 *$ \\
\hline & & Reação vivencial - ME & $0,18 * * *$ \\
\hline & & Emotividade - ME & $0,07 * * *$ \\
\hline \multirow[t]{3}{*}{ Modelo 7} & \multirow[t]{3}{*}{ Recompensas por colaboração espontânea } & Tônus.vital - MD & $0,20 * *$ \\
\hline & & Agressividade - MD & $0,15 * * *$ \\
\hline & & Predomínio - tensional - ME & $0,18 * * *$ \\
\hline \multirow[t]{2}{*}{ Modelo 8} & \multirow[t]{2}{*}{ Recompensas por observância às normas } & Predomínio tensional - MD & $0,15^{* * *}$ \\
\hline & & Tônus vital - ME & $0,19 * *$ \\
\hline Modelo 9 & $\begin{array}{l}\text { Recompensas por desempenho eficaz } \\
\text { e eficiente na execução das funções } \\
\text { do cargo }\end{array}$ & Tônus vital - MD Agressividade - MD & $\begin{array}{l}0,16^{* * *} \\
0,17 * * *\end{array}$ \\
\hline
\end{tabular}

Nota. Legenda: TV: Tônus vital; AGR: Agressividade; RV: Reação vivencial; EMO: Emotividade; PRE TEN: Predomínio tensional; DIM TEN: Dimensão tensional; MD: Mão direita; ME: Mão esquerda.

$* p<0,01 ; * * 0,01<p<0,05 ; * * * 0,05<p<0,25$.

da medida de desviance $(\Delta-2 \mathrm{LL} ; p<0,05)$. No entanto, os valores globais da significância estatística dos modelos não foram satisfatórios. Por fim, os coeficientes $R^{2} \operatorname{Cox}$ Snell e $R^{2}$ Nagelkerke apresentaram valores próximos de 0 , e a medida de desviance (-2LL) foi superior ao valor de referência de 1 . A interpretação dessas três medidas indica que os modelos não representam adequadamente a associação das dimensões de personalidade e o desempenho no trabalho. Logo, com base nos parâmetros de avaliação de modelos, pode-se concluir que as variáveis explicativas inseridas em cada um dos modelos não foram preditoras adequadas da variação observada na medida de desempenho no trabalho.

\section{Discussão}

Os resultados das análises da associação entre as dimensões do PMK em relação ao desempenho indicaram que as variáveis comunicação disciplinar e recompensa, e as respectivas subcategorias, apresentaram associações significativas com as medidas de personalidade. No entanto, a magnitude dessas relações foi fraca e não se sustentaram na análise de regressão logística. Esses resultados indicaram que as dimensões do PMK, isoladas ou em conjunto, não estiveram associadas ao desempenho no trabalho.

Desse modo, a hipótese do estudo não foi confirmada. Ou seja, não houve associação entre controle sobre a 
expressão emocional e comportamental, índices de tônus vital e agressividade variando entre normal a aumentado e desempenho no trabalho (recompensas e comunicações disciplinares). Em que pese a utilização de técnicas multivariadas com a finalidade de contemplar a complexidade dos construtos personalidade e desempenho no trabalho, conforme sugerido por Schmidt e Hunter (1998) e Tett e Christiansen (2007), as associações entre as medidas foram nulas.

Algumas considerações podem ser levantadas tanto em relação às medidas preditoras quanto às de critério utilizadas. Em relação às primeiras, os resultados indicam a necessidade de se utilizar as dimensões de personalidade obtidas por meio do PMK com cautela como base para a tomada de decisão na seleção de pessoas na organização estudada. Conforme já apresentado, na literatura brasileira, há apenas o estudo quantitativo desenvolvido por Baumgartl e Primi (2006) em que se investigou a associação das dimensões do PMK com o desempenho no trabalho (A. G. Vasconcelos, 2010). Os resultados do estudo foram convergentes com aqueles aqui apresentados e indicam a necessidade de mais estudos para avaliar a adequação do PMK, em termos de validade preditiva, considerando-se os contextos de utilização do teste e a carência de fundamentação teórica do instrumento que, até então, se limita as considerações propostas por Mira y Lopes (Sisto, 2010; A. G. Vasconcelos, 2010). Em relação a esse aspecto, considera-se importante a atualização dos princípios teóricos apresentados pelo autor que embasam o instrumento sob a perspectiva dos avanços teóricos e metodológicos da Psicologia. Diante de tais resultados, conclui-se que, do ponto de vista aplicado, estudos de validade são necessários para legitimar o uso do PMK no contexto de seleção de pessoas, uma vez que as evidências existentes na literatura são escassas.

Quanto às medidas de desempenho (presença de recompensas e comunicações disciplinares), os critérios foram categorizados de acordo com os registros disponíveis na organização. As quatro categorias de recompensa foram, a saber, apresentar desempenho eficaz e eficiente por meio do emprego de capacidade técnica em benefício do serviço, colaborar de maneira espontânea com a efetividade dos resultados pretendidos, participar em eventos e observar as normas e as prescrições regulamentares. Já as comunicações disciplinares foram classificadas em cinco categorias (faltar ou chegar atrasado ao serviço injustificadamente o que compromete a execução dos serviços e atividades, apresentar desempenho insuficiente na execução das tarefas, apresentar postura desrespeitosa, negligenciar o cumprimento de normas, prazos e tarefas, ter postura incompatível com os princípios e valores da organização). Nota-se que as medidas de critério utilizadas foram consonantes com a proposta de Borman e Motowidlo (1997) já que se tratavam de aspectos referentes ao desempenho contextual. No entanto, a adoção desses critérios não contribuiu para a força das associações. Por se tratar de um estudo de caso, sugere-se que a organização realize um aprimoramento dos critérios de desempenho no trabalho. Será um diferencial a identificação de critérios de desempenho do cargo empiricamente associados com as medidas de personalidade. Caso a opção seja o desenvolvimento de uma avaliação de desempenho, sugere-se realizar treinamentos com os avaliadores a fim de que ofereçam notas consistentes aos avaliados, influenciadas minimamente por aspectos subjetivos.

\section{Considerações Finais}

Há um interesse em compreender os processos psicológicos subjacentes ao desempenho no trabalho. Em especial, busca-se desenvolver teorias e técnicas de avaliação capazes de oferecer informações objetivas que reduzam a incerteza na tomada de decisão. Na seleção de pessoas, a principal justificativa para a utilização dos testes psicológicos é a capacidade preditiva das medidas em relação a um critério de desempenho subsequente no trabalho. No entanto, os profissionais devem reconhecer que existem limites no grau em que tais critérios podem ser preditos. A tomada de decisão na seleção de pessoas está sujeita aos erros de mensuração observados nos testes psicológicos como também ao fato do comportamento no trabalho ser influenciado pela interação de uma variedade de fatores organizacionais, sociais e também outras variáveis individuais não contemplados no exame psicológico para a admissão. Por isso, qualquer característica psicológica avaliada não pode ser considerada como um preditor preciso e isolado do desempenho no trabalho.

Ressalta-se que, apesar dos resultados obtidos em relação às dimensões do PMK, não há uma argumentação a favor do abandono do construto personalidade como potencial preditor do desempenho no trabalho. Tett e Christiansen (2007) enumeram procedimentos que podem auxiliar na melhoria da habilidade preditiva dos traços de personalidade em relação ao desempenho, tais como: (a) análise consistente do cargo considerando-se variáveis individuais e organizacionais, (b) delimitação de traços específicos nos modelos preditivos e, (c) verificação estatística da interação entre os traços e outras características psicológicas nos modelos hipotetizados.

Nesse sentido, as contribuições de Schmidt e Hunter (1998) são relevantes para evidenciar a importância de se considerar a validade incremental de medidas de personalidade em relação às medidas de habilidade cognitiva geral em contextos e funções específicos. Traços psicológicos podem ser elementos importantes para oferecer validade incremental para a predição do desempenho, em especial fatores Integridade e Conscienciosidade. De acordo com os autores, indivíduos mais conscienciosos tendem a buscar maior domínio da função, e, consequentemente, obter melhores desempenhos, pois se empenham e gastam mais tempo na tarefa do que pessoas menos conscienciosas. Ademais, os autores identificaram três combinações entre 
traços de personalidade e habilidade cognitiva que, em geral, apresentam altos índices de validade e utilidade. As medidas de validade preditiva do desempenho obtida a partir da combinação das medidas de habilidade cognitiva geral e os testes de integridade oferecem um poder preditivo médio ( $R$ múltiplo) de 0,65 . Já a combinação composta pelos escores dos testes cognitivos e medidas de amostra de trabalhadores para candidatos que já dominam a função para qual se candidatam oferece poder preditivo médio $(R$ múltiplo) de 0,63 . Por fim, os autores apontam que a combinação de testes cognitivos a entrevistas estruturadas para avaliar Conscienciosidade, Amabilidade, Neuroticismo, conforme o modelo dos cinco fatores, juntamente com provas de conhecimento em relação a aspectos do trabalho pode ser a indicada, uma vez que a validade preditiva atinge um valor igual a 0,63 .

De acordo com essas perspectivas de pesquisa sobre instrumentos de autorrelato, percebe-se a relevância de dar continuidade aos estudos que investigam a validade dos modelos preditivos das medidas de personalidade em relação ao desempenho no trabalho, em especial dos testes expressivos, como o PMK. A relação entre esses construtos deve ser melhor compreendida. Morgeson et al. (2007b) e Sarason, Sarason e Pierce (1995) afirmam que seria um entendimento simplista desconsiderar a influência dos traços de personalidade, uma vez que o mau desempenho do indivíduo no trabalho não pode ser explicado por dificuldades cognitivas. Nesse sentido, estes autores incentivam a continuidade da pesquisa a fim de desenvolver bases teóricas que esclareçam a associação entre os traços psicológicos e o desempenho.

Conclui-se que os psicólogos que trabalham com a avaliação psicológica no contexto do trabalho ainda enfrentam dificuldades de ordem técnica e legal. Esta investigação, em consonância com o Conselho Federal de Psicologia (CFP, 2003) e SIOP (2003), evidencia a importância de pesquisas recorrentes para acumular evidências de validade dos testes psicológicos. O desenvolvimento de estudos de validade relacionada a critério na seleção de pessoas tende a oferecer grande contribuição para que as medidas psicológicas e também o trabalho do psicólogo nesse campo de atuação se consolidem como fontes de informação legítimas e confiáveis para a tomada de decisão relativa à admissão de candidato na organização.

Logo, constata-se a necessidade de que esforços sejam destinados ao desenvolvimento de pesquisas que investiguem a capacidade preditiva dos instrumentos psicológicos utilizados na seleção de pessoas em relação ao desempenho subsequente no cargo, em especial relativos ao PMK (Sisto, 2010). Caso se julgue necessário, é importante ainda a construção de novos instrumentos e adaptação, validação e normatização dos já existentes para os contextos específicos com o intuito de obter resultados confiáveis.

\section{Referências}

American Educational Research Association, American Psychological Association, \& National Council on Measurement in Education. (1999). Standards for educational and psychological testing. Washington, DC: American Educational Research Association.

Ayçaguer, L. C. S., \& Utra, I. M. B. (2004). Regressión logística. Cuardenos de estadística. Madri, España: La Muralla.

Baumgartl, V. O., \& Primi, R. (2006). Contribuições da avaliação psicológica no contexto organizacional: Um estudo com o BPR-5, o BFM-1 e o PMK. São Paulo, SP: Casa do Psicólogo.

Binning, J. F., \& Barrett, G. V. (1989). Validity of personnel decisions: A conceptual analysis of the inferential and evidential bases. Journal of Applied Psychology, 74(3), 478-494.

Borman, W. C., \& Modowidlo, S. J. (1997). Task performance and contextual performance: The meaning for personnel selection research. Human Performance, 10(2), 99-109.

Conselho Federal de Psicologia. (2003). Resolução CFP $n^{o}$ 002/2003. Retirado de http://www.pol.org.br

Cronbach, L. J. (1949). Essentials of psychological testing. New York: Harper.

Cronbach, L. J., \& Meehl, P. E. (1955). Construct validity in psychological tests. Psychological Bulletin, 52, 281-302.

Dias, M. (2005). Absenteísmo em contact center: Estudo de caso com preditores micro e macro-organizacionais (Dissertação de mestrado, Universidade Federal de Uberlândia, Uberlândia, MG, Brasil).

Embretson, S. E. (1983). Construct validity: Construct representation versus nomothetic span. Psychological Bulletin, 93(1), 179-197.

Freitas, L. C. O. (2004). Avaliação psicológica em concurso público: Relações com o desempenho em treinamento de bombeiros (Dissertação de mestrado, Universidade de Brasília, DF, Brasil).

Galland de Mira, A. M. (2002). PMK: Psicodiagnóstico Miocinético. São Paulo, SP: Vetor.

Hosmer, D. W., \& Lemeshow, S. (2000). Applied logistic regression. New York: John Willy.

Hough, L. M., \& Oswald, F. L. (2000). Personnel selection. Annual Review of Psychology, 51, 631-664.

Hunter, J. E., \& Hunter, R. F. (1984). Validity and utility of alternative predictors of job performance. Psychological Bulletin, 96(1), 72-98.

Kuncel, N. R., Hezlett, S. A., \& Ones, D. S. (2004). Academic performance, career potential, creativity, and job performance: Can one construct predict them all? [Special section]. Journal of Personality and Social Psychology, 86, 148-161.

Morgeson, F. P., Campion, M. A., Dipboye, R. L., Hollenbeck, J. R., Murphy, K., \& Schimtt, N. (2007a). Reconsidering use of personality tests in personnel selection contexts. Personnel Psychology, 60, 683-729.

Morgeson, F. P., Campion, M. A., Dipboye, R., Hollenbeck, J. R, Murphy, K., \& Schmitt, N. (2007b). Are we getting fooled again? Coming to terms with limitations in the use of personality tests for personnel selection. Personnel Psychology, 60, 1029-1049.

Motowidlo, S. J., Borman, W. C., \& Schmit, M. J. (1997). A theory of individual differences in task and contextual performance. Human Performance, 10(2), 71-83.

Mount, M. K., Barrick, M. R., \& Strauss, J. P. (1999). The joint relationship of conscientioness and ability with performance: Test of the interaction hypothesis. Journal of Management, 25(5), 707-716. 
Robertson, I. T., \& Smith, M. (2001). Personnel selection. Journal of Occupational and Organizational Psychological, 74, 441-472.

Salgado, J. F. (1997). The five factor model of personality and job performance in the European community. Journal of Applied Psychology, 82, 30-43.

Sampaio, J. R. (1998). Testes psicológicos no processo de seleção de pessoal. In I. B. Goulart \& J. R. Sampaio (Eds.), Psicologia do trabalho e gestão de recursos humanos: Estudos contemporâneos. São Paulo, SP: Casa do Psicólogo.

Sarason, I. G., Sarason, B. R., \& Pierce, G. R. (1995). Cognitive interference at the intelligence-personality crossroads. In D. H. Saklofske \& M. Zeider (Eds.), International handbook of personality and intelligence. New York: Plenum Press.

Schmidt, F. L., \& Hunter, J. E. (1998). The validity and utility of selection methods in personnel psychology: Practical and theoretical implications of 85 years of research findings. Psychological Bulletin, 124(2), 262-274.

Schmidt, F. L., \& Hunter, J. E. (2004). General mental ability in the world of work: Occupational attainment and job performance. Journal of Personality and Social Psychology, 86(1), 162-173.

Schmitt, N., \& Landy, F. J. (1993). The concept of validity. In N. Schmitt, W. C. Borman, \& Society for Industrial and Organizational Psychology, Personnel selection in organizations. São Francisco, CA: Jossey-Bass.

Siegel, S., \& Castellan, N. J., Jr. (2006). Estatística não-paramétrica para ciências do comportamento (2. ed.). Porto Alegre, RS: Artmed.

Sisto, F. F. (2010). Uma análise das evidências de validade brasileiras do PMK. Psico USF, 15(2), 141-149.

Smith, M. (1994). A theory oh the validity of predictors in selection. Journal of Occupational and Organizational Psychology, 67(1), 13-19.

Society for Industrial and Organizational Psychology. (2003). Principles for the validation and use of personnel selection procedures. Retrieved from http://www.siop.org

Spector, P. (2004). Psicologia nas organizações. São Paulo, SP: Saraiva.

Tett, R. P., \& Christiansen, N. D. (2007). Personality tests at the crossroads: A response to Morgeson, Campion, Dipboye, Hollenbeck, Murphy and Schimtt (2007). Personnel Psychology, 60, 967-993.

Vasconcelos, A. G. (2010). Evidências de validade preditiva de medidas psicológicas em relação ao desempenho no trabalho: Um estudo de caso em uma organização militar (Dissertação de mestrado, Programa de Pós-Graduação em Psicologia, Universidade Federal de Minas Gerais, Belo Horizonte, MG, Brasil).

Vasconcelos, A. G., Nascimento, E., \& Sampaio, J. R. (2011). Validade preditiva do $\mathrm{PMK}$ em relação à presença de sintomas psicopatológicos. Psico USF, 16(2), 143-149.

Vasconcelos, T. S. (2005). O Inventário Fatorial dos Cinco Fatores de Personalidade no ambiente de trabalho (Tese de doutorado. Universidade de Brasília, DF, Brasil). 